\title{
Pedestrian tracking from a moving vehicle
}

\author{
Vasanth Philomin, Ramani Duraiswami, and Larry Davis \\ Computer Vision Laboratory \\ Institute for Advanced Computer Studies \\ University of Maryland, College Park, MD 20742, USA \\ \{vasi,ramani,lsd\}@cs.umd.edu
}

\begin{abstract}
Intelligent vehicles and unattended driving systems of the future will need the ability to recognize relevant traffic participants (such as other vehicles, pedestrians, bicyclists, etc.) and detect dangerous situations ahead of time. An important component of these systems is one that is able to distinguish pedestrians and track their motion to make intelligent driving decisions. The associated computer vision problem that needs to be solved is detection and tracking of pedestrians from a moving camera, which is extremely challenging. Robust pedestrian tracking performance can be achieved by temporal integration of the data in a probabilistic setting. We employ a shape model for pedestrians and an efficient variant of the Condensation tracker to achieve these objectives. The tracking is performed in the high-dimensional space of shape model parameters which consists of Euclidean transformation parameters and deformation parameters. Our Condensation tracker employs sampling on quasirandom points, improving its asymptotic complexity and robustness, and making it amenable to realtime implementation.
\end{abstract}

Keywords Driver Assistance Systems, Active Safety, Condensation Tracker, Linear Shape Models, Quasi-random Sampling

\section{Introduction}

There has been a growing interest in the automotive/transportation industry to make sensor-based systems to assist drivers for safety and comfort enhancement. A concerted effort is currently underway at Daimler-Chrysler to extend vision-based navigation beyond the highway scenario into the complex urban environment [4]. The application is difficult because it involves a complex outdoor environment (e.g. illumination conditions, unpredictable interaction between traffic participants, cluttered background), which cannot be controlled. Objects to be detected in this scenario have a large variety of appearances due to different shapes, sizes, orientation with respect to the camera, etc. Furthermore, we have a moving camera, so simple background subtraction techniques do not apply. Finally, we have strong limitations on the computational costs of methods when considering the speed of the moving vehicle. However, on-board automotive driver assistance systems that inform/alert a driver about the current speed, an inadmissible turn or collision with other traffic participants, are no longer just a dream.

The problem of pedestrian detection has been addressed in [5] and [9], but without temporal integration of results. We believe that temporal integration of results is essential for the demanding performance rates that might be required for the actual deployment of such a system. Furthermore, the use of temporal data enables one to make predictions about the motion of the pedestrian, and estimate probabilities of collision. This tracking problem, however, is complicated because there is significant camera motion, and pedestrians move according to unpredictable/unknown motion models. We want to make no assumptions about how the camera is moving (translation, rotation, etc.) or about the viewing angle. Hence it is not practically feasible to break up the dynamics (motion model) into several different motion classes $([2,11])$ and learn the dynamics of each class and the class transition probabilities. We need a general model that is able to cope with the wide variety of motions exhibited by both the camera and the pedestrian, as well as with the shape variability of the pedestrian being tracked.

This paper is organized as follows: Section 2 gives a brief introduction to the shape model used to model pedestrians and explains how to learn the model automatically from segmented pedestrian contours. This gives a set of deformation parameters which along with the Euclidean parameters (translation, rotation and scaling) constitute the state space. Section 3 describes a novel tracking algorithm that addresses the issues of an unknown motion model, robustness to outliers, and use of quasi-random points for efficiency. In Section 4 we successfully apply this algorithm to real video sequences of pedestrians. Section 5 concludes the paper. 


\section{Learning a linear pedestrian model}

The Point Distribution Model (PDM) [3] has proven to be a useful method for building a compact linear shape model from training examples of a class of shapes. The conventional PDM requires manual labelling of a set of points called the "landmark" points in each training image. These points are concatenated to form a shape vector and the shape vectors resulting from all the training images are aligned using Procrustes analysis [6]. A mean shape and a set of modes of variation are then generated using Principal Component Analysis (PCA). A method for automatically extracting the human silhouettes from a training set of images and building a linear shape model is described in [1]. First, the silhouettes are extracted using background subtraction followed by morphological operations and then tracing the boundary points of the resulting foreground regions to form edge chains. A uniform B-spline with the control points placed at approximately uniformly spaced intervals along the contour is produced efficiently from each of these silhouettes. The control points of the B-spline are then used as the landmark points in the PDM.

We use techniques similar to that described in [1] and [3] with some improvements to build a linear pedestrian model. One improvement is in the parameterization of the B-spline curve that is fitted to each extracted contour. Suppose that the set of points in a single pedestrian contour is $\left\{Q_{k}\right\}, k=$ $0, \ldots, m$, and we want to approximate these points with a $p^{t h}$ degree B-spline. Suppose that the values for the parameters $\bar{u}_{k}$ and the knot vector $U=$ $u_{0}, \ldots, u_{r}$ are precomputed and known. We then set up and solve the (unique) linear least squares problem for the unknown control points $P_{i}$. Assume that $p \geq 1, m>n$ and $n \geq p$. We seek a $p^{t h}$ degree nonrational curve

$$
C(u)=\sum_{i=0}^{n} N_{i, p}(u) P_{i} \quad u \in[0,1]
$$

satisfying:

- $Q_{0}=C(0)$ and $Q_{m}=C(1)$;

- the remaining $Q_{k}$ are approximated in the least squares sense, i.e.

$$
\sum_{k=1}^{m-1}\left|Q_{k}-C\left(\bar{u}_{k}\right)\right|^{2}
$$

is a minimum with respect to the $n+1$ variables, $P_{i}$; the $\left\{\bar{u}_{k}\right\}$ are the precomputed parameter values and $N_{i, p}$ are the $p^{t h}$ degree $\mathrm{B}$-spline basis functions. The resulting curve generally does not pass precisely through $Q_{k}$, and $C\left(\bar{u}_{k}\right)$ is not the closest point on $C(u)$ to $Q_{k}$.

The choice of $\bar{u}_{k}$ and $U$ affects the shape and parameterization of the curve. The most common method for choosing $\bar{u}_{k}$ is the chord length parameterization, which is the one used in [1]. Here, if $d$ is the total chord length given by

$$
d=\sum_{k=1}^{m}\left|Q_{k}-Q_{k-1}\right|
$$

then $\bar{u}_{0}=0, \bar{u}_{m}=1$ and

$$
\bar{u}_{k}=\bar{u}_{k-1}+\frac{\left|Q_{k}-Q_{k-1}\right|}{d} \quad k=1, \ldots, m-1
$$

This gives a good parameterization of the curve in the sense that it approximates a uniform parameterization. However, when the data takes very sharp turns such as in the case of pedestrian shapes, the chord length method does not perform well. We use the centripetal method ([8]) that gives better results with such data, where if

$$
d=\sum_{k=1}^{m} \sqrt{\left|Q_{k}-Q_{k-1}\right|}
$$

then $\bar{u}_{0}=0, \bar{u}_{m}=1$ and

$$
\bar{u}_{k}=\bar{u}_{k-1}+\frac{\sqrt{\left|Q_{k}-Q_{k-1}\right|}}{d} \quad k=1, \ldots, m-1
$$

The placement of the knots should reflect the distribution of the $\left\{\bar{u}_{k}\right\}$ and we choose the knot vector $U$ as follows. Let $c=\frac{m+1}{n-p+1}$, then the internal knots are given by

$$
\begin{gathered}
i=\lfloor j c\rfloor \quad \alpha=j c-i \\
u_{p+j}=(1-\alpha) \bar{u}_{i-1}+\alpha \bar{u}_{i} \quad j=1, \ldots, n-p
\end{gathered}
$$

Equation 1 guarantees that every knot span contains at least one $\bar{u}_{k}$, and under this condition the matrix in the least squares formulation is positive definite and well-conditioned.

We also use a weighted least squares method to align two shapes in the Procrustes analysis, where the weights are chosen so that more significance is given to the more stable landmark points i.e. the points which vary their position the least over the entire training set. As a result, emphasis is given to aligning the stable parts of the object rather than the unstable parts during shape alignment. Figure 1 shows some of the significant modes of variation of the pedestrian shapes in the training set. 


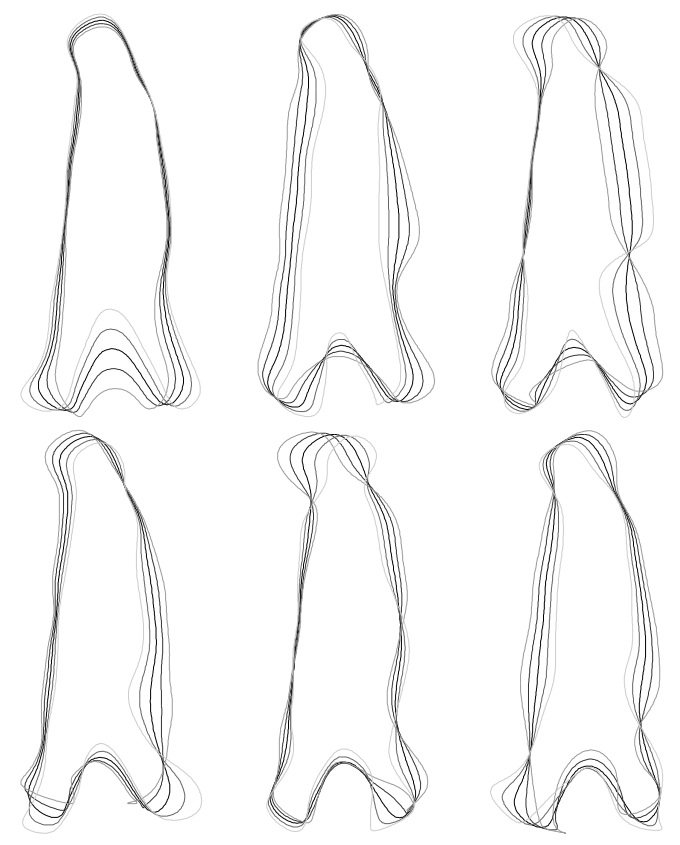

Figure 1: Modes of variation of pedestrian shapes

\section{Tracking algorithm}

The Condensation algorithm [7] has attracted much interest in the active vision area as it offers a framework for dynamic state estimation where the underlying probability density functions (pdfs) need not be Gaussian. The algorithm is based on a Monte Carlo or sampling approach, where the pdf is represented by a set of random samples. As new information becomes available, the posterior distribution of the state variables is updated by recursively propagating these samples (using a motion model as a predictor) and resampling. An accurate dynamical model is essential for robust tracking and for achieving real-time performance. This is due to the fact that the process noise of the model has to be made artificially high in order to track objects that deviate significantly from the learned dynamics, thereby increasing the extent of each predicted cluster in state space. One would then have to increase the sample size to populate these large clusters with enough samples. A high-dimensional state space (required for tracking complex shapes such as pedestrians) only makes matters worse. Even when one uses a "perfect" pseudo-random sequence for generating $N$ sample points, the sampling error will only decrease as $O\left(N^{-1 / 2}\right)$ as opposed to $O\left(N^{-1}\right)$ for another class of sequences known as quasi-random sequences which have low discrepancy. We introduced quasi-random sampling in the context of the Condensation algorithm in Philomin et al. [10] and showed that even in low dimensions, a significantly fewer amount of sample points were needed to achieve the same sampling error when compared to pseudo-random sampling. For reasons of brevity, the details are not discussed here; the readers are referred to [10]. In typical implementations of the Condensation algorithm, a "perfect" pseudorandom number generator is almost never used and a linear congruential generator (such as the system supplied rand()function) is used instead. These generators, although very fast, have an additional inherent weakness that they are not free of sequential correlation on successive calls, i.e. if $k$ random numbers at a time are used to generate points in $k$-dimensional space, the points will lie on $(k-1)$-dimensional planes and will not fill up the $k$-dimensional space.

Since we do not want to make any assumptions about how the vehicle and the pedestrian are moving or about the viewing angle, we propose using a zero-order motion model with large process noise high enough to account for the greatest expected change in shape and motion. In other words, we need to concentrate our samples in large regions around highly probable locations from the previous time step. These high-dimensional regions which correspond to the large process noise can now be efficiently sampled using quasi-random sampling as described below.

Given the sample set $\left\{\left(\mathbf{s}_{t-1}^{(n)}, \pi_{t-1}^{(n)}\right)\right\}$ at the previous time step, $\pi_{t-1}^{(n)}$ being the associated probabilities, we first choose a base sample $\mathbf{s}_{t-1}^{(i)}$ with probability $\pi_{t-1}^{(i)}$. This yields a small number of highly probable locations, say $M$, the neighborhoods of which we must sample more densely. If there were just one region requiring a dense concentration, an invertible mapping from a uniform space to the space of equal importance could be constructed, as given below in Equation (3) for the case of a multidimensional Gaussian. Since we have $M$ regions, the importance function cannot be constructed in closed form. One therefore needs an alternative strategy for generating from the quasi-random distribution, a set of points that samples important regions densely.

We have devised a simple yet effective strategy that achieves these objectives. Let the $M$ locations have centers $\mu^{(j)}$ and variances $\sigma^{(j)}$ based on the process noise, where these quantities are $k$ dimensional vectors. We then overlay $M+1$ distributions of quasi-random points over the space, with the first $M$ distributions made Gaussian, centered at $\mu^{(j)}$ and with diagonal variance $\sigma^{(j)}(3)$. Finally, we also overlay a $(M+1)$ th distribution that is spread uniformly over the entire state space. This provides robustness against sudden changes in shape and motion. The total number of points used is $N$, where

$$
N=N_{1}+N_{2}+\ldots+N_{M+1}
$$


the sample size in the Condensation algorithm. We have in effect chosen $\mathbf{s}_{t}^{(n)}$ by sampling from $p\left(\mathbf{X}_{t} / \mathbf{X}_{t-1}=\mathbf{s}_{t-1}^{(i)}\right)$.

The conversion from a uniform quasi-random distribution to a Gaussian quasi-random distribution is achieved using the mapping along the $l$ th dimension

$$
y_{j l}=\mu_{l}^{(j)}+\sqrt{2} \sigma_{l}^{(j)} \operatorname{erf}^{-1}\left(\left(2 \xi_{l}-1\right)\right),
$$

where $\operatorname{erf}^{-1}$ is the inverse of the error function given by

$$
\operatorname{erf}(z)=\frac{2}{\sqrt{\pi}} \int_{0}^{z} e^{-t^{2}} d t
$$

and $\xi_{l}$ represents the quasi-randomly distributed points in $[0,1]$.

Finally, we measure and compute the probabilities $\pi_{t}^{(n)}=p\left(\mathbf{Z}_{t} / \mathbf{X}_{t}=\mathbf{s}_{t}^{(n)}\right)$ for these new sample positions in terms of the image data $\mathbf{Z}_{t}$. We use a measurement density based on the multi-feature distance transform algorithm (see [5] for details) that has been successfully used for detecting pedestrians from static images. Therefore

$$
\begin{aligned}
\log p\left(\mathbf{Z}_{t} / \mathbf{X}_{t}\right)= & \log p(\mathbf{Z} / \mathbf{X}) \\
& \propto\left\{-\frac{1}{M} \sum_{i=1}^{M} d_{\text {typed }}^{2}\left(z_{i}, I\right)\right\},
\end{aligned}
$$

where the $z_{i}$ 's are measurement points along the contour, $I$ is the image data, and $d_{\text {typed }}\left(z_{i}, I\right)$ denotes the distance between $z_{i}$ and the closest feature of the same type in $I$. We use oriented edges discretized into eight bins as the features in all our experiments.

\section{Results}

We now present some results on tracking pedestrians from a moving vehicle using the techniques discussed above. First, a statistical shape model of a pedestrian was built using automatically segmented pedestrian contours from sequences obtained by a stationary camera (so that we can do background subtraction). We use the techniques described in Section 2 to build a LPDM (Linear Point Distribution Model). The dimensionality is then reduced by using Principal Component Analysis (PCA) to find an eight-dimensional space of deformations. Hence, the total dimension of $x_{t}$ (the state variable) is 12 (4 for the Euclidean similarity parameters and 8 for the deformation parameters). We used $N=2000$ samples and the tracker was initialized in the first frame of the sequence using the pedestrian detection algorithm described in [5]. We introduced $10 \%$ of random samples at every iteration to account for sudden changes in shape and motion. We applied the tracker to several pedestrian sequences and found that the quasi-random tracker was able to successfully track the pedestrians over the entire sequence. The tracker was also able to recover very quickly from failures due to sudden changes in shape or motion or to partial occlusion. On the other hand, the pseudo-random tracker was easily distracted by clutter and was unable to recover from some failures. Figure 2 shows the tracker output (both the modal (highest probability) state and the mean state) for a typical pedestrian sequence using the quasi-random tracker.

\section{Conclusions}

In this paper, we have developed a framework for tracking pedestrians from moving vehicles. Our method is based on the use of a linear shape model for the pedestrians, and temporally integrating data from multiple frames to achieve robustness. Our approach used the Condensation tracker, and extended it to high-dimensional problems by incorporating quasi-Monte Carlo methods into the conventional algorithm. We have also addressed the problem of making the tracker work efficiently in situations where the motion models are unknown. Future work will address tracking multiple people, and real-time implementations of this system.

\section{References}

[1] A. Baumberg and D. C. Hogg. Learning flexible models from image sequences. In Proc. European Conference on Computer Vision, 1994.

[2] A. Blake, B. North and M. Isard. Learning multi-class dynamics. In Advances in Neural Information Processing Systems, 11, 1999. in press.

[3] T. F. Cootes, C. J. Taylor, A. Lanitis, D. H. Cooper and J. Graham. Building and using flexible models incorporating grey-level information. In Proc. IEEE International Conference on Computer Vision, pages 242246, 1993.

[4] U. Franke, D. Gavrila, S. Goerzig, F. Lindner, F. Paetzhold and C. Woehler. Autonomous driving approaches downtown. to appear in IEEE Expert (special issue on Vision-based Driving Assistance in Vehicles of the Future), 1997.

[5] D. Gavrila and V. Philomin. Real-time object detection for "smart" vehicles. In Proc. IEEE 

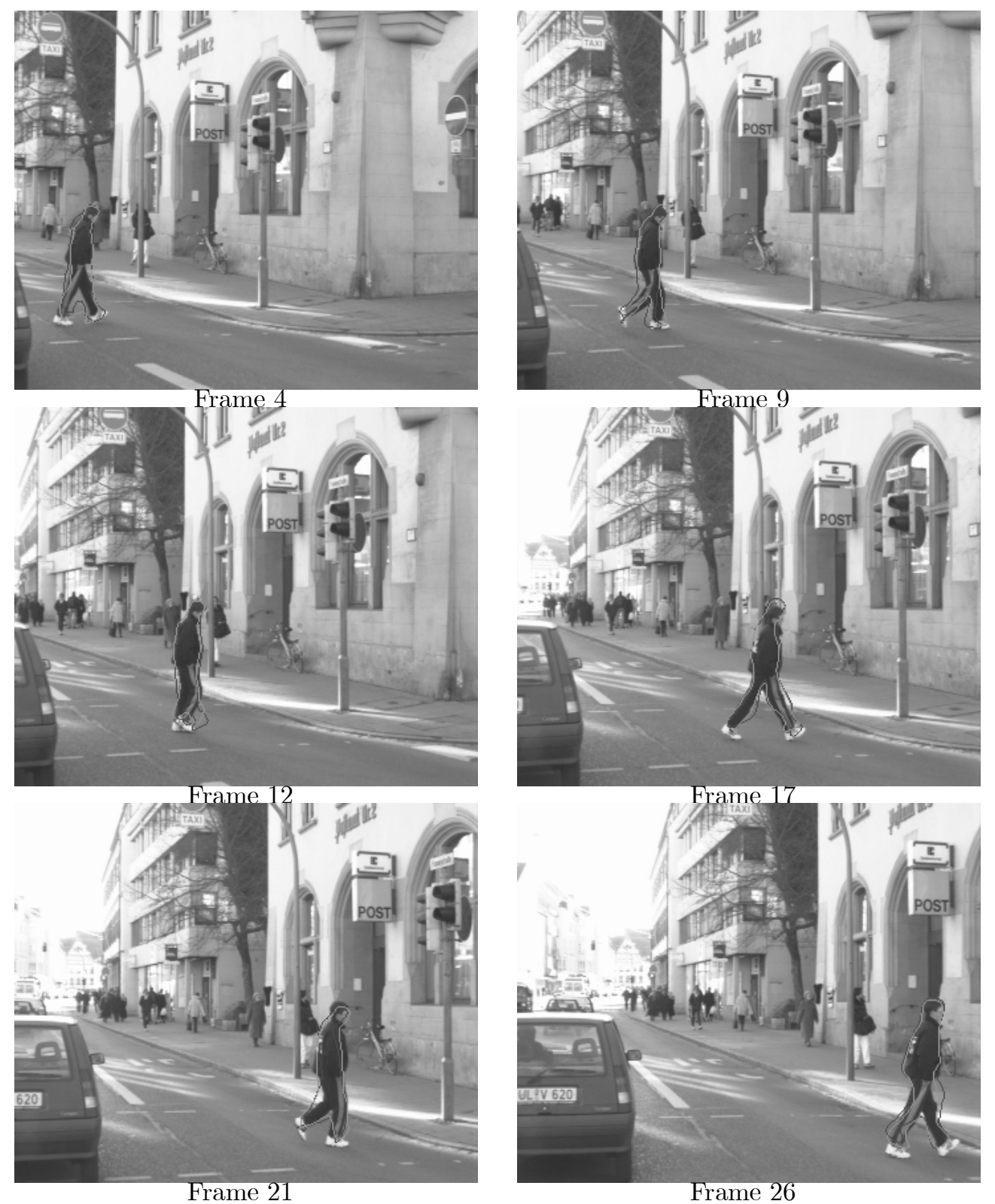

Figure 2: Tracking results for Daimler-Chrysler pedestrian sequence using quasi-random sampling. Dark - Highest probability state estimate; Light - Mean state estimate. 
International Conference on Computer Vision, Volume 1, pages 87-93, Kerkyra, Greece, 1999.

[6] C. Goodall. Procrustes methods in the statistical analysis of shape. Journal of the Royal Statistical Society B, Volume 53, Number 2, pages 285-339, 1991.

[7] M. Isard and A. Blake. Contour tracking by stochastic propagation of conditional density. In Proc. European Conference on Computer Vision, pages 343-356, Freiburg, Germany, 1996.

[8] E. T. Y. Lee. Choosing nodes in parametric curve interpolation. In $C A D$, Volume 21, pages 363-370, 1989.

[9] M. Oren, C. Papageorgiou, P. Sinha, E. Osuna and T. Poggio. Pedestrian detection using wavelet templates. In Proc. IEEE International Conference on Computer Vision, pages 193-199, 1997.

[10] V. Philomin, R. Duraiswami and L. S. Davis. Quasi-random sampling for condensation. In Proc. European Conference on Computer Vision, 2000.

[11] J. Rittscher and A. Blake. Classification of human body motion. In Proc. IEEE International Conference on Computer Vision, pages 634-639, 1999. 